\title{
Physical, chemical and toxicological properties of polycyclic aromatic hydrocarbons (PAHs) in human exposure assessments to contaminated soil and groundwater
}

\author{
Alexandru Balint $^{1 *}$ \\ ${ }^{1}$ University of Bucharest, Doctoral School of Geology, 6 Traian Vuia, Bucharest, Romania
}

\begin{abstract}
Polycyclic aromatic hydrocarbons (PAHs) are a class of chemical substances consisting of carbon and hydrogen only, which have multiple fused ring systems. Although PAHs are widely recognised as contaminants of concern ( $\mathrm{CoC})$, no applicable limits have been established for groundwater in the Romanian legislation. Furthermore, the existing methodologies do not include guidelines for assessing the human exposure to contaminated soil and groundwater. The current research aims at identifying the relevant physical, chemical and toxicological properties of PAHs when conducting human exposure assessments for contaminated and potentially contaminated sites. The methods applied in the research are based on the general geoenvironmental risk assessment procedure by analysing the sources potentially generating PAHs, the migration and exposure pathway depending on their fate and transport properties, and the vulnerability to PAHs of the potential receptors, based on their toxicological profile.
\end{abstract}

\section{Introduction}

The Romanian legislation on contaminated sites is undergoing a significant updating process which started in 2018 with the new Law no. 74/2019 on the management of potentially contaminated sites and contaminated sites. The risk assessment is defined in the Romanian legislation through the Government Emergency Ordnance no. 195/2005 as the report prepared by authorized natural or legal persons which analyzes the probability and severity of the main components of the environmental impact and establishes the need for prevention, intervention and/or remedial measures.

There are many available methods and tools which may be suitable for risk assessments, the current paper analyses polycyclic aromatic hydrocarbons (PAH) for summarizing their main parameters which should be taken into consideration during a risk assessment, especially when focusing on the exposure of receptors to contaminated soil and groundwater.

\footnotetext{
${ }^{*}$ Corresponding author: ioan.alexandru.balint@,drd.unibuc.ro
} 
Although the article focuses on several specific risk assessment standards, the parameters are suitable for use in any type of risk assessment if they are adapted to the specific geological and hydrogeological conditions of the sites which are assessed.

\section{General information}

\subsection{Petroleum hydrocarbons}

Petroleum hydrocarbons are a general class of compounds which consist almost entirely of carbon and hydrogen. Petroleum hydrocarbons may be classified into two large categories: aromatics and aliphatic.

The general classification of petroleum hydrocarbons is included in Figure 1.

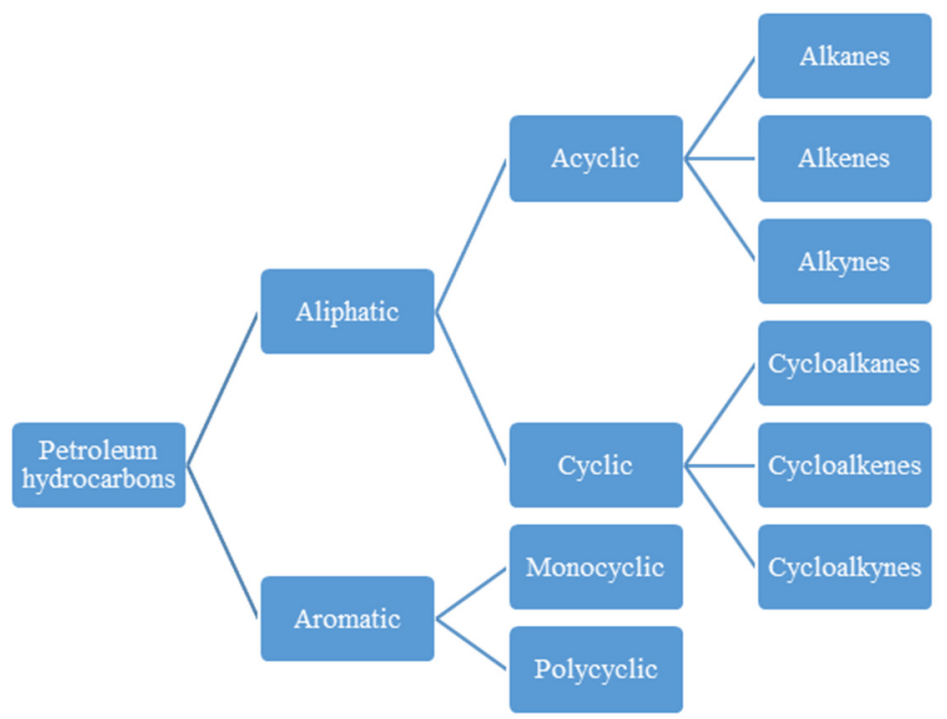

Fig. 1. General classification of petroleum hydrocarbons.

The definition of each category is included below [1]:

- Alkanes are saturated acyclic hydrocarbons consisting entirely of single carbon-carbon bonds s type. The general formula of alkanes is $\mathrm{C}_{n} \mathrm{H}_{2 n+2}$ and examples include methane $\left(\mathrm{CH}_{4}\right)$, ethane $\left(\mathrm{C}_{2} \mathrm{H}_{6}\right)$, propane $\left(\mathrm{C}_{3} \mathrm{H}_{8}\right)$, butane $\left(\mathrm{C}_{4} \mathrm{H}_{10}\right)$ etc.

- Alkenes are acyclic hydrocarbons which have one carbon-carbon double bond. The general formula of alkenes is $C_{n} H_{2 n}$ and examples include ethene/ethylene $\left(\mathrm{C}_{2} \mathrm{H}_{4}\right)$, propene $\left(\mathrm{C}_{3} \mathrm{H}_{6}\right)$, butene $\left(\mathrm{C}_{4} \mathrm{H}_{8}\right)$ etc. Depending on the number of double bonds, the class of hydrocarbons may be referred to as alkadienes (two double bonds), alkatrienes (three double bonds).

- Alkynes are acyclic hydrocarbons which have one carbon-carbon triple bond. The general formula of alkynes is $\mathrm{C}_{n} \mathrm{H}_{2 n-2}$ and examples include ethyne/ acetylene $\left(\mathrm{C}_{2} \mathrm{H}_{2}\right)$, propyne/ methylacetylene $\left(\mathrm{C}_{3} \mathrm{H}_{4}\right)$, butyne $\left(\mathrm{C}_{4} \mathrm{H}_{6}\right)$ etc.

- Cycloalkanes/alkenes/alkynes are cyclic hydrocarbons in which the first and last carbon atoms in the hydrocarbon chain are connected. Cyclic hydrocarbons should not be confused with aromatic hydrocarbons detailed below.

- Aromatic cyclic hydrocarbons which follow the Hückel $(4 n+2)$ rule derived from the Hückel molecular orbital (HMO) theory which states that "monocyclic planar systems of trigonally hybridized atoms that contain $(4 n+2) \pi$-electrons, where $\mathrm{n}$ is a positive integer, 
will exhibit aromatic character" [1]. It is widely accepted that aromatic hydrocarbons are substances which contain one benzene ring as in the case of monocyclic compounds (e.g. benzene, toluene, ethylbenzene and o-, m-, p-xylenes, also known as BTEX), or more than one benzene ring as in the case of polycyclic compounds (e.g. naphthalene, phenanthrene).

\subsection{Behavior of petroleum hydrocarbons}

The main specific categories of contaminants from petroleum hydrocarbons depending on their fate and transport in soil and groundwater include volatile organic compounds (VOCs), light non-aqueous phase liquids (LNAPLS), dense non-aqueous phase liquids (DNAPLs).

Volatile organic compounds (VOCs) are organic compounds that evaporate at room temperature, which are also referred to as solvents, although solvents are a class of VOCs used in coatings, as cleaning agents etc. The European Directive 2010/75/EU on industrial emissions, defines VOCs are any organic compound having at $293.15 \mathrm{~K}\left(20{ }^{\circ} \mathrm{C}\right)$, a vapor pressure of $0.01 \mathrm{kPa}$ or more. Generally, a higher vapour pressure indicates a higher volatility. VOCs can be released in the environment from various industries which include manufacture or use of paints and degreasing products, gasoline storage, septic systems etc. After reaching the unsaturated area, they may remain suspended as residuals or they might migrate upwards through the aerated pores or downward towards the groundwater [2-4].

Light non-aqueous phase liquids (LNAPLs) are organic compounds which are immiscible with water and have a density which is lower than $1 \mathrm{~kg} \cdot 1^{-1}$. Several LNAPLs such as BTEX, vinyl chloroform and tetrahydrofuran [5] are VOCs. Other LNAPL components will remain entrapped in the soil pores as residual LNAPLs or they will migrate vertically with a small horizontal expansion towards the saturated zone, depending on the water content of the soil pores and the potential energy of the LNAPLs. Mobile LNAPLs are usually located in the capillary fringe and/or at the top of the groundwater [4]. If the potential energy of the LNAPLs is sufficient to displace the water of the capillary fringe, they will migrate vertically until they will reach the saturated zone. In areas where the potential energy is insufficient to displace the pore water during vertical migration, the LNAPLs will begin a lateral migration in most cases [6].

Dense non-aqueous phase liquids (DNAPLs) are organic compounds which are immiscible with water and have a density which is higher than $1 \mathrm{~kg} \cdot \mathrm{l}^{-1}$. Like LNAPLs, several DNAPLs are also VOCs. Due to their high density, DNAPLs will migrate more easily than LNAPLs vertically and partially laterally towards the saturated zone. Once entering groundwater, DNAPLs tend to settle at the base of the aquifer and they are transported in the direction of the groundwater flow.

The most representative DNAPLs include polyaromatic hydrocarbons (PAHs), polychlorinated biphenyls (PCBs) and chlorinated solvents such as trichloroethylene (TCE) and tetrachloroethylene (PCE).

\subsection{Polycyclic aromatic hydrocarbons (PAHs)}

Polycyclic aromatic hydrocarbons (PAHs) are a class of chemical substances consisting of carbon and hydrogen only, which have multiple fused ring systems [7, 8].

There are more than 100 compounds classified as PAHs [9], although from a toxicological point of view, only 16 PAHs are frequently monitored in Europe and USA [10] and have been considered in the current article: acenaphthene, acenaphthylene, anthracene, benz[a]anthracene, benzo[b]fluoranthene, benzo[k]fluoranthene, benzo[g,h,i]perylene, benzo[a]pyrene, chrysene, dibenz[a,h]anthracene, fluoranthene, fluorene, indeno[1,2,3-cd]pyrene, naphthalene, phenanthrene and pyrene. All chemical 
substances may be easily identified based on their CAS registry number provided in the following sections.

\section{Materials and methods}

In its most basic form, risk is defined as a measure of uncertain future events and it has its origins in the probability theories developed in the $16^{\text {th }}$ and $17^{\text {th }}$ centuries [11]. Risk may be assessed starting from basic equations, such as the one provided by the US Environmental Protection Agency which defines risk as "the likelihood that adverse ecological effects may occur or are occurring because of exposure to one or more stressors" [12]:

One of the documents which is used by most risk assessors for petroleum contaminated sites is the American Standard ASTM E1739 - 95(2015) Standard Guide for Risk-Based Corrective Action Applied at Petroleum Release which provides several equations for quantitative risk assessments depending on the type of medium and exposure routes as follows: inhalation of contaminated air, ingestion of contaminated groundwater, inhalation of contaminated vapors from soil and, ingestion, inhalation of contaminated vapors and particulates as well as dermal contact from surficial contaminated soil and risks of leaching from contaminated soil to groundwater [13].

Prior to identifying parameters, a general conceptual site model was considered in accordance with the standard ASTM E1689-20 Standard Guide for Developing Conceptual Site Models for Contaminated Sites, which defines conceptual site models as arepresentation of "the biological, physical and chemical processes that determine the transport of contaminants from sources through environmental media to environmental receptors within the system" [14].

Based on the definition provided by the ASTM standard, any risk assessment should begin with a conceptual site model to identify the source of contamination, the migration pathway, the exposure routes, and the receptors.

\section{Results}

\subsection{Physical and chemical properties of PAHs}

There are multiple sources of PAHs since they are generated by thermal decomposition of organic materials which may include fossil fuel or biomass, landfill leachates as reported in the United States of America, Sweden and Poland at various concentrations and by industrial activities [15-17]. They can result from the energy industry, mining, transportation, or petrochemical industry.

Once PAHs are released in soil, they are biodegraded rapidly in an aerobic environment, but they may persist longer times under anaerobic conditions or in large concentrations, as they may be toxic to microorganisms [2]. If they are not biodegraded in soil, some tend to be adsorbed on soil surface based on their octanol-carbon coefficient $\left(\mathrm{K}_{\mathrm{oc}}\right)$, volatilize based on their vapor pressure from dry soil or from wet soil based on their Henry's law coefficient, while others tend to migrate towards groundwater.

After reaching the groundwater, several PAHs will tend to remain at the top of the water or migrate to the base of the aquifer depending on their density, others might dissolve in water depending on their octanol-water coefficient $\left(\mathrm{K}_{\text {ow }}\right)$ and water solubility and some PAHs tend to volatilize from water based on their Henry's law constant $(\mathrm{H})$ and solubility.

A general conceptual model was included in Figure 2 below. 


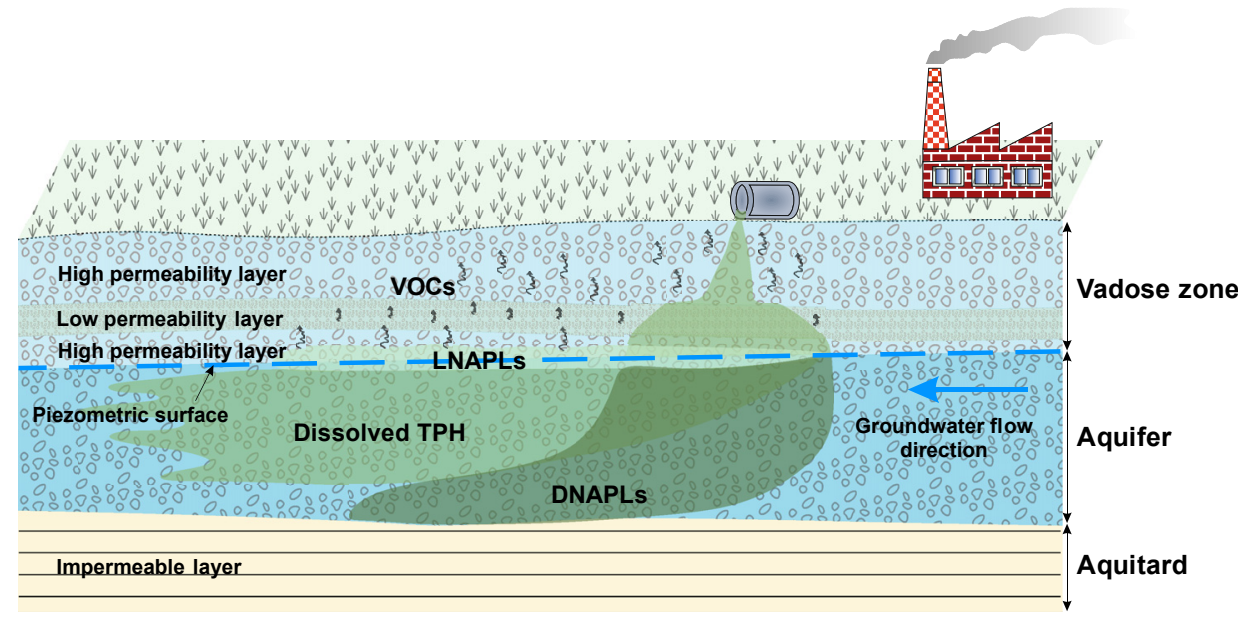

Fig. 2. Conceptual site model for the migration of petroleum hydrocarbons in soil and groundwater.

If the contaminants will reach a human receptor, mostly through the saturated media which provides higher transport potential on extended areas in comparison with the unsaturated media where the vertical migration component is dominant, exposure may occur through inhalation, ingestion or dermal contact during various household activities [4]. Figure 3 below is an example of exposure to PAHs which may occur around a landfill. An exposure assessment requires a focus on the receptors rather than a focus on the source of contamination, although a sound understanding of the source may improve the results of the exposure assessment.

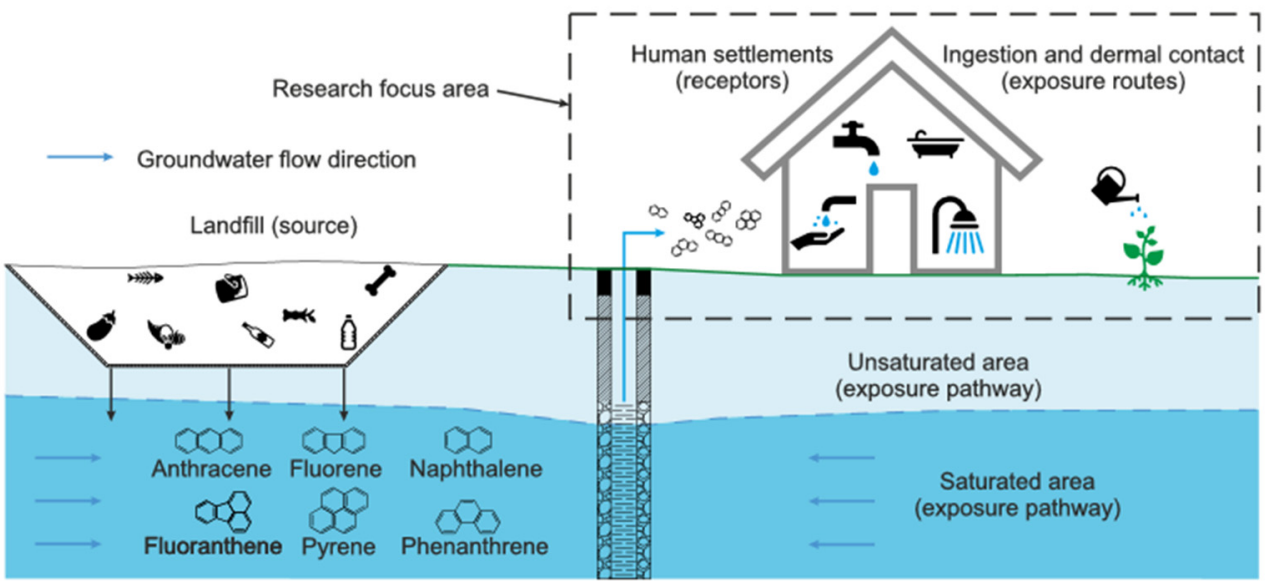

Fig. 3. Conceptual site model for exposure assessments.

The physical and chemical properties of PAHs were summarized from multiple authors and the results were included in Table 1 below. 


\begin{tabular}{|c|c|c|c|c|c|c|c|c|c|c|c|c|c|c|c|c|}
\hline 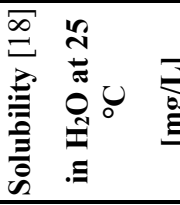 & $\stackrel{\mathrm{m}}{\mathrm{m}}$ & $\ddot{6}$ & $\begin{array}{l}\stackrel{+}{0} \\
\stackrel{0}{0} \\
\dot{0}\end{array}$ & $\begin{array}{l}\text { ¿े } \\
\dot{8} \\
\dot{0}\end{array}$ & & & $\begin{array}{l}c \\
0 \\
8 \\
8 \\
0\end{array}$ & 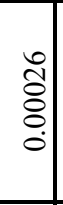 & $\begin{array}{l}\tau \\
8 \\
0 \\
0\end{array}$ & 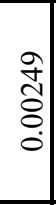 & 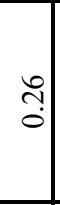 & 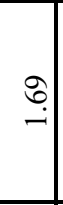 & $\begin{array}{l}0 \\
\overline{8} \\
8 \\
0 \\
0\end{array}$ & $\vec{m}$ & $\stackrel{n}{=}$ & $\begin{array}{l}n \\
m \\
0\end{array}$ \\
\hline 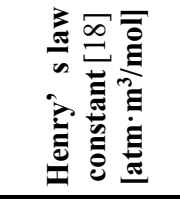 & $\begin{array}{l}\dot{0} \\
0 \\
\dot{+} \\
\infty \\
-i\end{array}$ & 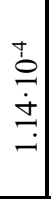 & $\begin{array}{l}3 \\
0 \\
\dot{0} \\
n \\
n\end{array}$ & $\begin{array}{l}n \\
0 \\
\dot{0} \\
\end{array}$ & & & $\begin{array}{l}\hat{0} \\
\dot{1} \\
\hat{i}\end{array}$ & $\begin{array}{l}\hat{0} \\
\dot{m} \\
m\end{array}$ & $\begin{array}{l}0 \\
\dot{0} \\
\dot{n} \\
\\
\text { in }\end{array}$ & $\begin{array}{l}\hat{0} \\
\dot{\vec{\sigma}} \\
\dot{\dot{\theta}}\end{array}$ & $\begin{array}{l}0 \\
\dot{0} \\
\dot{b} \\
\infty \\
\infty\end{array}$ & $\begin{array}{l}3 \\
\dot{0} \\
\dot{\hat{\sigma}} \\
a\end{array}$ & $\begin{array}{c}\hat{0} \\
\dot{\infty} \\
\dot{+} \\
\dot{r}\end{array}$ & $\begin{array}{l}\dot{0} \\
0 \\
\dot{b} \\
\dot{+}\end{array}$ & $\begin{array}{l}\tilde{0} \\
\dot{\hat{n}} \\
\stackrel{+}{+}\end{array}$ & $\begin{array}{l}n \\
0 \\
\dot{0} \\
= \\
-1\end{array}$ \\
\hline 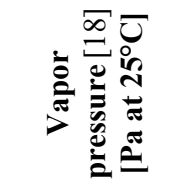 & సे & $\begin{array}{l}\partial \\
\dot{0}\end{array}$ & $\begin{array}{c}\hat{3} \\
0 \\
\dot{\vec{t}} \\
\infty\end{array}$ & $\begin{array}{l}0 \\
0 \\
\dot{0} \\
\infty \\
i\end{array}$ & & & $\begin{array}{l}\hat{0} \\
\dot{\tilde{n}} \\
\stackrel{?}{n}\end{array}$ & $\begin{array}{c}0 \\
\dot{0} \\
\dot{m} \\
\\
-1\end{array}$ & $\begin{array}{l}\hat{0} \\
\dot{\bar{m}} \\
\infty\end{array}$ & 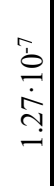 & $\begin{array}{c}\hat{0} \\
\dot{0} \\
\end{array}$ & $\begin{array}{l}1 \\
0 \\
\dot{0} \\
\infty \\
\end{array}$ & \begin{tabular}{c|}
$\infty$ \\
0 \\
$\ddots$ \\
$\dot{\sigma}$ \\
-1
\end{tabular} & $\stackrel{m}{=}$ & $\begin{array}{l}\hat{N} \\
\dot{\sigma} \\
\dot{\sigma} \\
-\end{array}$ & $\begin{array}{l}+ \\
0 \\
\dot{8} \\
\dot{0} \\
\dot{0}\end{array}$ \\
\hline $\begin{array}{l}3 \\
0 \\
0 \\
0 \\
0 \\
0 \\
0\end{array}$ & $\begin{array}{l}a \\
\varrho \\
2 \\
\dot{n}\end{array}$ & $\begin{array}{l}a \\
2 \\
2 \\
\infty \\
\dot{m}\end{array}$ & 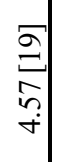 & $\begin{array}{l}\sigma \\
\Xi \\
\infty \\
\infty \\
i\end{array}$ & $\begin{array}{l}\sigma \\
2 \\
0 \\
0 \\
i\end{array}$ & $\begin{array}{l}2 \\
2 \\
2 \\
\infty \\
\dot{n}\end{array}$ & $\begin{array}{l}a \\
\stackrel{2}{3} \\
0 \\
0\end{array}$ & $\begin{array}{l}a \\
己 \\
0 \\
0\end{array}$ & 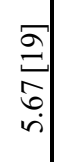 & 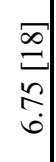 & $\begin{array}{l}a \\
\vdots \\
\hat{\sigma} \\
\dot{+}\end{array}$ & $\begin{array}{l}\Xi \\
\Xi \\
\Xi \\
\dot{\nabla}\end{array}$ & 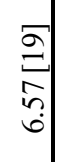 & $\begin{array}{l}a \\
\stackrel{a}{a} \\
\dot{9} \\
\dot{r}\end{array}$ & 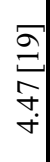 & $\begin{array}{l}a \\
\Xi \\
0 \\
i\end{array}$ \\
\hline 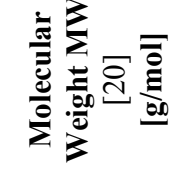 & 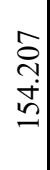 & 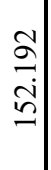 & 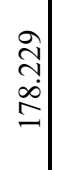 & 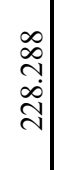 & 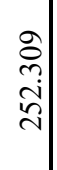 & 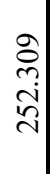 & 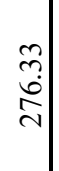 & $\begin{array}{l}8 \\
\text { సे } \\
\text { సે} \\
\text { ते }\end{array}$ & 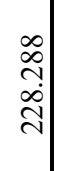 & $\begin{array}{c}0 \\
+ \\
\vdots \\
\infty \\
\hat{N}\end{array}$ & 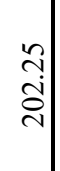 & 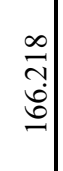 & 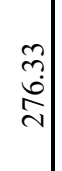 & 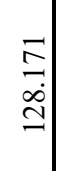 & 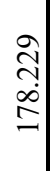 & 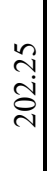 \\
\hline 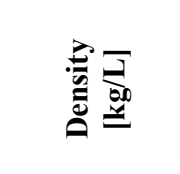 & 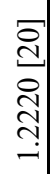 & 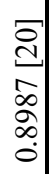 & 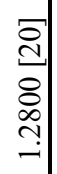 & 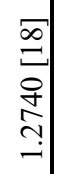 & 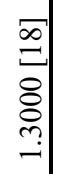 & 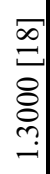 & 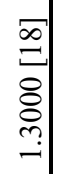 & 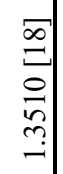 & 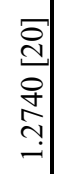 & 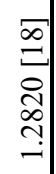 & 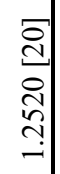 & 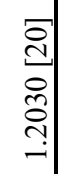 & 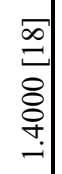 & 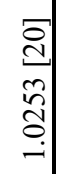 & 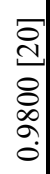 & 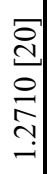 \\
\hline 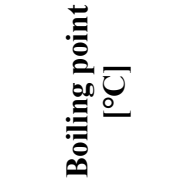 & 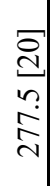 & 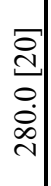 & 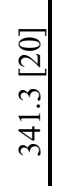 & 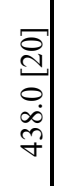 & 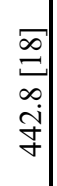 & 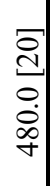 & 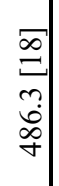 & 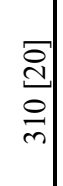 & $\begin{array}{l}\text { ㄹ. } \\
0 \\
0 \\
\dot{+}\end{array}$ & 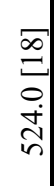 & 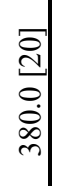 & 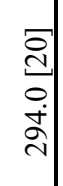 & $\begin{array}{l}\infty \\
\Xi \\
0 \\
0 \\
n \\
n\end{array}$ & $\begin{array}{l}\overrightarrow{0} \\
\stackrel{0}{0} \\
\dot{\infty} \\
\vec{v}\end{array}$ & 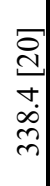 & 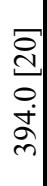 \\
\hline 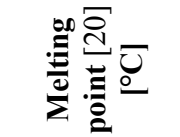 & $\hat{\sigma}$ & $\begin{array}{l}+ \\
\grave{\infty}\end{array}$ & $\stackrel{0}{\sim}$ & 잉 & $\begin{array}{l}+ \\
\dot{0} \\
0 \\
-1\end{array}$ & $\vec{\nabla}$ & $\begin{array}{l}\stackrel{0}{\infty} \\
\sim\end{array}$ & $\stackrel{શ}{\beth}$ & $\tilde{n}$ & 워 & $\begin{array}{l}\stackrel{1}{0} \\
ٍ \\
=\end{array}$ & $\begin{array}{l}\stackrel{2}{1} \\
\dot{\Xi} \\
\Xi\end{array}$ & ర్ర & 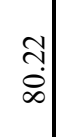 & ๙ & $\begin{array}{l}\text { రై } \\
\stackrel{\circ}{n}\end{array}$ \\
\hline 预 & $\stackrel{0}{\stackrel{0}{ت}}$ & 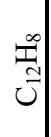 & 孛 & $\underset{\Xi}{\stackrel{\sim}{ \pm}}$ & 昰 & 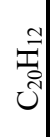 & 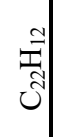 & $\underset{\widetilde{\Im}}{\Im}$ & 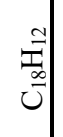 & |さ્ડ & |원 & 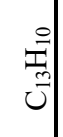 & $\underset{\widetilde{J}}{\stackrel{\Xi}{\Xi}}$ & $\begin{array}{l}\beth_{0}^{\infty} \\
\stackrel{0}{U}\end{array}$ & 丞 & \\
\hline 莺 & 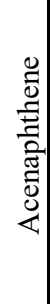 & 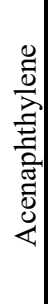 & 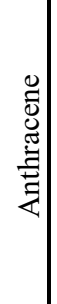 & 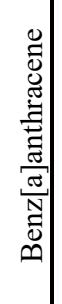 & 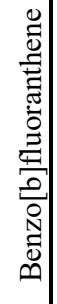 & 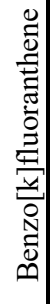 & $\begin{array}{l}0 \\
0 \\
0 \\
0 \\
0 \\
= \\
=00 \\
0 \\
0 \\
0 \\
0 \\
0\end{array}$ & $\begin{array}{l}0 \\
0 \\
0 \\
0 \\
0 \\
0 \\
0 \\
0 \\
0 \\
0\end{array}$ & $\begin{array}{l}0 \\
0 \\
0 \\
0 \\
0\end{array}$ & 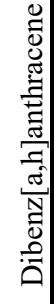 & 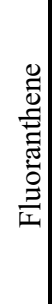 & 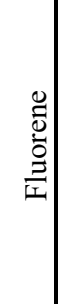 & $\begin{array}{l}0 \\
0 \\
0 \\
0 \\
0 \\
0 \\
0 \\
0 \\
\vdots \\
0 \\
0 \\
0 \\
0 \\
0\end{array}$ & 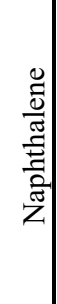 & 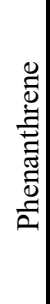 & 苟 \\
\hline
\end{tabular}




\subsection{Toxicological properties of PAHs}

The countries from the European Union are required to comply with the Regulation no. 1272/2008 of the European Parliament and of the Council of 16 December 2008 on classification, labelling and packaging of substances and mixtures, amending and repealing Directives 67/548/EEC and 1999/45/EC, and amending Regulation (EC) No 1907/2006, which contains a list of harmonized classification and labelling of hazardous substances in Part 3 of the regulation. Substances may be identified based on their Index no in accordance with the regulation, the EC number provided by the European Community or CAS no. provided by the Chemical Abstract Service.

In accordance with the Regulation no. 1272/2008, chemical substances and mixtures are classified based on their hazard class:

- Physical hazards: explosives, flammable gases, flammable aerosols, oxidizing gases, gases under pressure, flammable liquids, flammable solids, self-reactive substances and mixtures, pyrophoric liquids, pyrophoric solids, self-heating substances and mixtures, substances, and mixtures which in contact with water emit flammable gases, oxidizing liquids, oxidizing solids, organic peroxides and corrosive to metals.

- Health hazards: acute toxicity, skin corrosion/irritation, serious eye damage/eye irritation, respiratory or skin sensitization, germ cell mutagenicity, carcinogenicity, reproductive toxicity, specific target organ toxicity (STOT) - single exposure, specific target organ toxicity (STOT) - repeated exposure and aspiration hazard.

- Environmental hazards: hazardous to aquatic environment.

- Additional hazard classes: hazardous to ozone layer.

The toxicology of chemical substances may be expressed based on several parameters in human exposure assessments as follows:

- Minimal Risk Levels (MRLs) provided by the US Agency for Toxic Substances and Disease Registry (ATSDR), which are defined as "an estimate of the amount of a chemical a person can eat, drink, or breathe each day without a detectable risk to health", if the substances are not carcinogenic.

- Oral reference dose $\left(\mathrm{RfD}_{\mathrm{o}}\right)$ and inhalation reference concentration (RfC) established by the US Environmental Protection Agency [18] based on the no-observed-adverse-effect level (NOAEL) and uncertainty factor (UF).

- The International Agency for Research on Cancer (IARC) classifies agents in four categories of carcinogens:

- Group 1: carcinogenic to humans when there is sufficient evidence of carcinogenicity in humans and animals.

- Group 2A: probably carcinogenic to humans when two of the following evaluations were made by the working group and at least one of them involves humans or human cells/tissues - there is limited evidence of carcinogenicity in humans, sufficient evidence of carcinogenicity in animals and strong evidence that the agent exhibits key characteristic of carcinogens.

- Group 2B: possibly carcinogenic to humans when only one evaluation was performed by the working group in conformity with the requirements under group 2A.

- Group 3: not classifiable as to its carcinogenicity to humans when the agent may not be classified in previous categories.

- The oral slope factor $\left(\mathrm{SF}_{\mathrm{o}}\right)$ which is an estimate of the increased cancer risk from oral exposure to a dose of $1 \mathrm{mg} / \mathrm{kg}$-day for a lifetime.

- The inhalation slope factors $\left(\mathrm{SF}_{\mathrm{i}}\right)$ derived from the inhalation unit risk (IUR) which is an estimate of the increased cancer risk from inhalation exposure to a concentration of 1 $\mu \mathrm{g} / \mathrm{m}^{3}$ for a lifetime.

The toxicological values for each of the 16 PAHs were included in Table 2 below. 
Table 2. Toxicological values and classifications for PAHs relevant for risk and exposure assessments.

\begin{tabular}{|c|c|c|c|c|c|c|c|c|c|c|c|c|}
\hline \multirow{3}{*}{ Chemical name } & & \multirow{3}{*}{$\begin{array}{c}\text { Reg. } \\
1272 / 2008\end{array}$} & \multicolumn{8}{|c|}{ Toxic data } & \multicolumn{2}{|c|}{$\begin{array}{c}\text { Carcinogenic } \\
\text { data }\end{array}$} \\
\hline & & & \multicolumn{3}{|c|}{$\begin{array}{c}\text { MRLs [24] } \\
\text { Oral - O }\left[\mathbf{m g}^{-1} \cdot \mathbf{k g}^{-}\right. \\
\left.\text {'day }^{-1}\right] \\
\text { Inhalation - I } \\
{[\mathrm{ppm}]}\end{array}$} & \multicolumn{3}{|c|}{$\begin{array}{c}\mathbf{R f D}_{\mathbf{0}}[18] \\
{\left[\mathbf{m g} \cdot \mathbf{k g}^{-1} \cdot \mathbf{d a y}^{-1}\right]}\end{array}$} & \multirow{2}{*}{\begin{tabular}{|} 
IUR \\
{$[18]$} \\
{$[(\mu \mathbf{g} \cdot$} \\
$\left.\left.\mathbf{m}^{-3}\right)^{-1}\right]$
\end{tabular}} & \multirow{2}{*}{$\begin{array}{l}\text { RfC- }- \\
\text { chron } \\
\text { ic }[18] \\
{[\mathbf{m g} \cdot} \\
\left.\mathbf{m}^{-3}\right]\end{array}$} & \multirow{2}{*}{$\begin{array}{c}\text { IAR } \\
\text { C } \\
\text { Class }\end{array}$} & \multirow{2}{*}{\begin{tabular}{|}
$\mathbf{S F}_{\mathbf{0}}$ \\
{$[18]$} \\
{$[\mathbf{m g} \cdot \mathbf{k}$} \\
$\mathbf{\mathbf { g } ^ { - }}$ \\
${ }^{1 \cdot \mathbf{d a y}}$ \\
$\left.{ }^{1}\right]$
\end{tabular}} \\
\hline & . & & $\left|\begin{array}{c}\text { Acut } \\
\text { e }\end{array}\right|$ & $\begin{array}{c}\text { Inter } \\
\text { m. }\end{array}$ & $\begin{array}{c}\text { Chron } \\
\text { ic }\end{array}$ & \begin{tabular}{|c|} 
Shor \\
$t-$ \\
term \\
\end{tabular} & $\begin{array}{c}\text { Subchro } \\
\text { nic }\end{array}$ & $\begin{array}{c}\text { Chron } \\
\text { ic }\end{array}$ & & & & \\
\hline Acenaphthene & $\begin{array}{c}a \\
\hat{d} \\
\hat{1} \\
\tilde{\infty}\end{array}$ & - & - & O: 0.6 & - & 0.6 & 0.2 & 0.06 & - & - & \begin{tabular}{|c|} 
Grou \\
p 3 \\
[21]
\end{tabular} & - \\
\hline Acenaphthylene & $\mid \begin{array}{l}1 \\
0 \\
a \\
0 \\
o \\
\end{array}$ & - & - & - & - & - & - & - & - & - & N/A & - \\
\hline Anthracene & 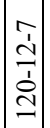 & - & - & O: 10 & - & 10 & 1 & 0.3 & - & - & \begin{tabular}{|c|} 
Grou \\
p 3 \\
{$[21]$}
\end{tabular} & - \\
\hline Benz[a]anthracene & $\begin{array}{l}n \\
\hat{n} \\
\hat{n} \\
\vdots \\
b \\
n\end{array}$ & $\begin{array}{c}\text { Carcinoge } \\
\text { nic 1B } \\
\text { Aquatic } \\
\text { acute 1 } \\
\text { Aquatic } \\
\text { Chronic 1 }\end{array}$ & - & - & - & - & - & - & $6 \times 10^{-5}$ & - & $\begin{array}{l}\text { Grou } \\
\text { p 2B } \\
{[21]}\end{array}$ & 0.1 \\
\hline $\begin{array}{c}\text { Benzo[b]fluoranth } \\
\text { ene }\end{array}$ & 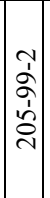 & $\begin{array}{c}\text { Carcinoge } \\
\text { nic 1B } \\
\text { Aquatic } \\
\text { acute } 1 \\
\text { Aquatic } \\
\text { Chronic 1 }\end{array}$ & - & - & - & - & - & - & $6 \times 10^{-5}$ & - & \begin{tabular}{|l|} 
Grou \\
p 2B \\
{$[21]$}
\end{tabular} & 0.1 \\
\hline $\begin{array}{c}\text { Benzo[k]fluoranth } \\
\text { ene }\end{array}$ & 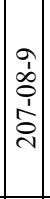 & $\begin{array}{c}\text { Carcinoge } \\
\text { nic 1B } \\
\text { Aquatic } \\
\text { acute } 1 \\
\text { Aquatic } \\
\text { Chronic 1 } \\
\end{array}$ & - & - & - & - & - & - & $6 \times 10^{-6}$ & - & \begin{tabular}{|l|} 
Grou \\
p 2B \\
{$[21]$}
\end{tabular} & 0.01 \\
\hline $\begin{array}{c}\text { Benzo[g,h,i]peryle } \\
\text { ne }\end{array}$ & $\begin{array}{c}\mathfrak{1} \\
\mathfrak{1} \\
\mathfrak{2} \\
\mathfrak{2}\end{array}$ & - & - & - & - & - & - & - & - & - & \begin{tabular}{|c|} 
Grou \\
p 3 \\
{$[21]$}
\end{tabular} & - \\
\hline Benzo[a]pyrene & $\mid \begin{array}{c}\infty \\
1 \\
\sim \\
\hat{1} \\
i \\
n\end{array}$ & $\begin{array}{c}\text { Carcinoge } \\
\text { nic } 2 \\
\text { Mutagenic } \\
2 \\
\text { Reproducti } \\
\text { ve toxicity } \\
2 \\
\end{array}$ & - & - & - & - & - & $3 \times 10^{-4}$ & $6 \times 10^{-4}$ & $2 \times 10^{-6}$ & $\begin{array}{c}\text { Grou } \\
\text { p } 1 \\
{[21,2} \\
2]\end{array}$ & 1 \\
\hline
\end{tabular}




\begin{tabular}{|c|c|c|c|c|c|c|c|c|c|c|c|c|}
\hline \multirow{3}{*}{ Chemical name } & & \multirow{3}{*}{$\begin{array}{c}\text { Reg. } \\
1272 / 2008\end{array}$} & \multicolumn{8}{|c|}{ Toxic data } & \multicolumn{2}{|c|}{$\begin{array}{c}\text { Carcinogenic } \\
\text { data }\end{array}$} \\
\hline & & & \multicolumn{3}{|c|}{$\begin{array}{c}\text { MRLs [24] } \\
\text { Oral - O [mg } \text {. }^{-} \\
\left.{ }^{1} \cdot \mathrm{day}^{-1}\right] \\
\text { Inhalation - I }^{-} \\
{[\mathrm{ppm}]}\end{array}$} & \multicolumn{3}{|c|}{$\begin{array}{c}\mathbf{R f D}_{\mathbf{0}}[18] \\
{\left[\mathbf{m g} \cdot \mathbf{k g}^{-1} \cdot \mathbf{d a y}^{-1}\right]}\end{array}$} & \multirow{2}{*}{\begin{tabular}{|} 
IUR \\
{$[18]$} \\
I $(\mathbf{\mu g} \cdot$ \\
$\left.\left.\mathbf{m}^{-3}\right)^{-1}\right]$
\end{tabular}} & \multirow{2}{*}{$\begin{array}{l}\text { RfC } \\
\text { chron } \\
\text { ic }[18] \\
{[\mathbf{m g} \cdot} \\
\left.\mathbf{m}^{-3}\right]\end{array}$} & \multirow{2}{*}{$\begin{array}{l}\text { IAR } \\
\text { C } \\
\text { Class }\end{array}$} & \multirow{2}{*}{\begin{tabular}{|c}
$\mathbf{S F}_{\mathbf{0}}$ \\
{$[18]$} \\
{$[\mathbf{m g} \cdot \mathbf{k}$} \\
$\mathbf{\mathbf { g } ^ { - }}$ \\
$\mathbf{1} \cdot \mathbf{d a y}$ \\
$\left.{ }^{\mathbf{1}}\right]$
\end{tabular}} \\
\hline & 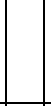 & & $\begin{array}{c}\text { Acut } \\
\text { e }\end{array}$ & $\begin{array}{c}\text { Inter } \\
\text { m. }\end{array}$ & $\begin{array}{c}\text { Chron } \\
\text { ic }\end{array}$ & \begin{tabular}{|c} 
Shor \\
t- \\
term
\end{tabular} & $\begin{array}{c}\text { Subchro } \\
\text { nic }\end{array}$ & $\begin{array}{c}\text { Chron } \\
\text { ic }\end{array}$ & & & & \\
\hline Chrysene & 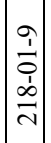 & $\begin{array}{c}\text { Carcinoge } \\
\text { nic } 2 \\
\text { Mutagenic } \\
3\end{array}$ & - & - & - & - & - & - & $6 \times 10^{-7}$ & - & $\begin{array}{l}\text { Grou } \\
\text { p 2B } \\
{[21]}\end{array}$ & 0.001 \\
\hline $\begin{array}{l}\text { Dibenz[a,h]anthra } \\
\text { cene }\end{array}$ & $\begin{array}{c}\hat{n} \\
\vdots \\
1 \\
\hat{n}\end{array}$ & $\begin{array}{c}\text { Carcinoge } \\
\text { nic } 2\end{array}$ & - & - & - & - & - & - & $6 \times 10^{-4}$ & - & $\begin{array}{l}\text { Grou } \\
\text { p 2A } \\
{[21]}\end{array}$ & 1 \\
\hline Fluoranthene & 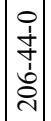 & - & & O: 0.4 & & 0.4 & 0.1 & 0.04 & - & - & $\begin{array}{c}\text { Grou } \\
\text { p } 3 \\
{[21]}\end{array}$ & - \\
\hline Fluorene & $\begin{array}{l}\hat{1} \\
\hat{2} \\
\hat{b} \\
\infty\end{array}$ & - & & O: 0.4 & & 0.4 & 0.4 & 0.04 & - & - & $\begin{array}{c}\text { Grou } \\
\text { p } 3 \\
{[21]}\end{array}$ & - \\
\hline $\begin{array}{l}\text { Indeno[1,2,3- } \\
\text { cd]pyrene }\end{array}$ & $\begin{array}{c}n \\
2 \\
\hat{2} \\
\hat{2} \\
2\end{array}$ & - & - & - & - & - & - & - & $6 \times 10^{-5}$ & - & $\begin{array}{l}\text { Grou } \\
\text { p 2B } \\
{[21]}\end{array}$ & 0.1 \\
\hline Naphthalene & $\begin{array}{c}0 \\
\hat{N} \\
\dot{N} \\
\alpha\end{array}$ & $\begin{array}{c}\text { Carcinoge } \\
\text { nic } 2 \\
\text { Acute } \\
\text { toxicity } 4 \\
\text { Aquatic } \\
\text { acute } 1 \\
\text { Aquatic } \\
\text { Chronic } 1 \\
\end{array}$ & $\begin{array}{c}\text { O: } \\
0.6\end{array}$ & O: 0.6 & $\begin{array}{c}\mathrm{I}: \\
7 \times 10^{-4}\end{array}$ & 0.6 & 0.6 & 0.02 & $\begin{array}{c}3.4 \times 1 \\
0^{-5}\end{array}$ & $3 \times 10^{-3}$ & $\begin{array}{l}\text { Grou } \\
\text { p 2B } \\
{[23]}\end{array}$ & 0.12 \\
\hline Phenanthrene & $\left|\begin{array}{c}\infty \\
1 \\
0 \\
1 \\
1 \\
\infty\end{array}\right|$ & - & - & - & - & - & - & - & - & - & $\begin{array}{c}\text { Grou } \\
\text { p } 3 \\
{[21]}\end{array}$ & - \\
\hline Pyrene & $\mid \begin{array}{c}0 \\
1 \\
\grave{1} \\
1 \\
\grave{1}\end{array}$ & - & - & - & - & - & 0.3 & 0.03 & - & - & $\begin{array}{c}\text { Grou } \\
\mathrm{p} 3^{\mathrm{c}}\end{array}$ & - \\
\hline
\end{tabular}




\section{Conclusions}

The melting points of PAHs suggest that they can be found in a solid state in the natural environment, but also in a liquid state if heated to approximately $100{ }^{\circ} \mathrm{C}$. Based on their boiling point between $218.0^{\circ} \mathrm{C}$ for naphthalene and $536.0^{\circ} \mathrm{C}$ for indeno[1,2,3-cd]pyrene, PAHs are not expected to evaporate through heating during normal household activities. As a result, if ingestion of contaminated groundwater would occur, the groundwater would not be suitable for consumption even after it is boiled.

The octanol-water partition coefficient suggests that all PAHs are highly hydrophobic, with lower values for acenaphthene, acenaphthylene and naphthalene. Based on the density of PAHs and their water solubility, it may be concluded that most of the chemicals behave as DNAPLs, except acenaphthylene and phenanthrene which behave as LNAPLs. Naphthalene has also a density which is closer to the density of water so accumulation at the base of the aquifer is expected to require more time than other PAHs.

The values of the vapor pressure suggest a low volatilisation rate from dry soil, with higher volatilisation rates for naphthalene and lower volatilisation rates for benzo[a]pyrene, while the Henry's law constant suggests that volatilisation may occur from water and wet soil with higher values for naphthalene and lower values for benzo[a]pyrene.

Based on the MRLs for PAHs, only one risk level of $0.6 \mathrm{mg} \cdot \mathrm{kg}^{-1} \cdot \mathrm{day}^{-1}$ and one of $7 \times 10^{-}$ ${ }^{4} \mathrm{mg} \cdot \mathrm{kg}^{-1} \cdot \mathrm{day}^{-1}$ were established for acute exposure (1-14 days) to naphthalene and chronic exposure ( $>365$ days), respectively. The lowest values for intermediate exposure ( $>14-364$ days) are $0.4 \mathrm{mg} \cdot \mathrm{kg}^{-1} \cdot \mathrm{day}^{-1}$ for exposure to fluoranthene and fluorene.

The lowest values for the oral reference dose are $0.4 \mathrm{mg} \cdot \mathrm{kg}^{-1} \cdot \mathrm{day}^{-1}$ for short-term exposure $\left(<30\right.$ days) to fluoranthene and fluorene, $0.1 \mathrm{mg} \cdot \mathrm{kg}^{-1} \cdot \mathrm{day}^{-1}$ for subchronic exposure (limited number of years, $<7$ years) to fluoranthene and $3 \times 10^{-4} \mathrm{mg} \cdot \mathrm{kg}^{-1} \cdot \mathrm{day}^{-1}$ for chronic exposure ( $>7$ years) to benzo[a]pyrene.

The highest values for the IUR are $6 \times 10^{-4}$ for benzo[a]pyrene and dibenz[a,h]anthracene, while the lowest $\mathrm{RfC}$ is $2 \times 10^{-6}$ for chronic exposure to benzo[a]pyrene.

Based on the IARC classification, benzo[a]pyrene was identified as being carcinogenic to humans, with a $\mathrm{SF}_{\mathrm{o}}$ of $1 \mathrm{mg} \cdot \mathrm{kg}^{-1} \cdot \mathrm{day}^{-1}$, dibenz[a,h]anthracene was identified as being probably carcinogenic for humans with a $\mathrm{SF}_{\mathrm{o}}$ of $1 \mathrm{mg} \cdot \mathrm{kg}^{-1} \cdot \mathrm{day}^{-1}$, while benz[a]anthracene, benzo[b]fluoranthene, benzo[k]fluoranthene, chrysene, indeno[1,2,3-cd]pyrene and naphthalene are possibly carcinogenic.

\section{References}

1. A.D. McNaught, and A. Wilkinson, Compendium of Chemical Terminology Gold Book (Blackwell Science, Oxford, 1997)

2. P. Wexler, et.al., Encyclopedia of Toxicology. Third Edition Volume 1 (Academic Press, Jamestown Road, 2014)

3. J.S. Zogorski, et.al., Volatile Organic Compounds in the Nation's Ground Water and DrinkingWater Supply Wells: U.S. Geological Survey Circular 1292 (U.S. Geological Survey, Denver, 2006)

4. C.W. Fetter, et.al. Contaminant Hydrogeology Third Edition (Waveland Press, Long Grove, 2018)

5. C.J. Newell, et.al. Light Nonaqueous Phase Liquids Report EPA/540/S-95-500 (US EPA, Washington DC, 1995)

6. CL:AIRE. An illustrated handbook of LNAPL transport and fate in the subsurface (CL:AIRE, London, 2014)

7. H.A. Favre, W.H. Powell, Nomenclature of Organic Chemistry: IUPAC Recommendations and Preferred Names 2013 (The Royal Society of Chemistry, Cambridge, 2014)

8. IUPAC. Compendium of Chemical Terminology: Gold Book (Blackwell Science, 2014) 
9. B.C. Crawford, B. Quinn, Microplastic Pollutants (Elsevier Science, 2017)

10. D. Lerda, Polycyclic Aromatic Hydrocarbons (PAHs) Factsheet 4th edition (European Commission - Joint Research Centre - Institute for Reference Materials and Measurements, 2011)

11. M. Weimer, European Journal of Risk Regulation 8 (1), 10-17 (2017)

12. EPA, Ecosystem Services as Assessment Endpoints in Ecological Risk Assessment (Technical Background Paper EPA/100/F-15/004, 2016)

13. ASTM E1739-95(2015) Standard Guide for Risk-Based Corrective Action Applied at Petroleum Release Sites (ASTM International, West Conshohocken, 2015)

14. ASTM E1689-20 Standard Guide for Developing Conceptual Site Models for Contaminated Sites, (ASTM International, West Conshohocken, 2020)

15. B.O. Clarke, et al., Chemosphere 127, 269-275 (2015)

16. T. Eggen, M. Moeder and A. Arukwe, Science of the Total Environment 408, 5147-5157 (2010)

17. R.H. Plumb, The Occurrence of Appendix IX Organic Constituents in Disposal Site Ground Water, 2, Groundwater Monitoring \& Remediation 11, 157-164 (1991)

18. US Department of Energy RAIS, RAIS Toxicity Values and Physical Parameters Search, The Risk Assessment Information System

19. Y-G. Ma, et al., J. Chem. Eng. 55 (2), 819-825 (2010)

20. W.M. Haynes, D.R. Lide and T.J. Bruno, CRC Handbook of Chemistry and Physics 97th edition, (CRC Press, Boca Raton, 2017)

21. IARC, IARC Monographs on the Evaluation of Carcinogenic Risks to Humans. Volume 92 Some Non-heterocyclic Polycyclic Aromatic Hydrocarbons and Some Related Exposures, (International Agency for Research on Cancer, Lyon, 2010)

22. IARC, IARC Monographs on the Evaluation of Carcinogenic Risks to Humans. Volume 100F A Review of Human Carcinogens, (International Agency for Research on Cancer, Lyon, 2012)

23. IARC, IARC Monographs on the Evaluation of Carcinogenic Risks to Humans. Volume 82 Some Traditional Herbal Medicines, Some Mycotoxins, Naphthalene and Styrene, (International Agency for Research on Cancer, Lyon, 2002)

24. MRLs Available on https://wwwn.cdc.gov/TSP/MRLS/mrlsListing.aspx 